\title{
Weak decays of doubly heavy baryons: Decays to a system of $\boldsymbol{\pi}$ mesons
}

\author{
A. S. Gerasimov ${ }^{1,2, *}$ and A. V. Luchinsky $\oplus^{1,3, \dagger}$ \\ ${ }^{1}$ NRC “Kurchatov Institute," IHEP, Protvino, Moscow Region, 142281, Russia \\ ${ }^{2}$ Moscow Institute of Physics and Technology, Institutskiy Pereulok, \\ 9, Dolgoprudny, Moscow Oblast, 141701, Russia \\ ${ }^{3}$ Bowling Green State University, Bowling Green, Ohio, 43402, USA
}

(Received 22 June 2019; published 30 October 2019)

\begin{abstract}
We consider exclusive weak decays of doubly heavy baryons with spin $J=1 / 2$ with production of a leptonic pair or a set of light mesons. Using the QCD factorization theorem and spectral functions approach, we obtain theoretical predictions for the partial probabilities of these decays and distributions over various kinematic variables. According to the obtained results, partial probabilities of some of the considered decays are large enough to be observed experimentally.
\end{abstract}

DOI: 10.1103/PhysRevD.100.073015

\section{INTRODUCTION}

Today the research of doubly heavy baryons takes a particularly important role in the physics of elementary particles. For a long time they were not available for experimental study. In 2005, the SELEX Collaboration reported [1] the observation of the $\Xi_{c c}^{+}$baryon in the decay $\Xi_{c c}^{+} \rightarrow p D^{+} K^{-}$. This result was not confirmed, but later the LHCb Collaboration managed to register the doubly heavy baryon $\Xi_{c c}^{++}$in the $\Lambda_{c}^{+} K^{-} \pi^{+} \pi^{+}$final state [2]. Thereby we are interested in theoretical research of other decays of doubly heavy baryons for subsequent experimental verification.

In this paper, decays of ground state doubly heavy baryons are considered: $\mathcal{B}_{1} \rightarrow \mathcal{B}_{2} R$, where $R=\mu \nu_{\mu}, \pi$, $2 \pi, 3 \pi, 5 \pi$. According to the factorization theorem they are related to $\tau$-lepton decays $\tau \rightarrow \nu_{\tau} R$, since in both cases the system $R$ is produced by the virtual $W$-boson transition $W \rightarrow R$. As a result, the probability of reaction $\mathcal{B}_{1} \rightarrow \mathcal{B}_{2} W$ is defined by the convolution of the semileptonic differential width $\mathcal{B}_{1} \rightarrow \mathcal{B}_{2} \mu \nu$ and spectral function of the transition $W \rightarrow R$. The first process can be described in terms of form factors of weak decay, which can be calculated with the help of potential models. Spectral functions, on the other hand, can be found by analyzing decays of the $\tau$-lepton $\tau \rightarrow \nu_{\tau} R$.

\footnotetext{
*Anton.Gerasimov@ihep.ru

†alexey.luchinsky@ihep.ru
}

Published by the American Physical Society under the terms of the Creative Commons Attribution 4.0 International license. Further distribution of this work must maintain attribution to the author(s) and the published article's title, journal citation, and DOI. Funded by SCOAP ${ }^{3}$.
The rest of the paper is organized as follows. In the next section, a description of the theoretical model used in our paper and parametrization of the form factor are given (see also the Appendix). Section III is devoted to a description of the spectral functions. In the next section we give our numerical results: integrated branching fractions of the considered decays and squared momentum distribution. Discussion of the obtained results is given in the final section of the paper.

\section{THEORETICAL DESCRIPTION}

Let us consider the reaction

$$
\mathcal{B}_{1} \rightarrow \mathcal{B}_{2} R
$$

where the doubly heavy baryon $\mathcal{B}_{1}$ with spin $J=1 / 2$ decays into another doubly heavy baryon $\mathcal{B}_{2}$ with the same spin and virtual $W$-boson, which then hadronizes into system $R$, where $R=\ell \nu_{\ell}, \pi, 2 \pi, 3 \pi, 5 \pi$.

The considered baryons contain heavy $b$ - and $c$-quarks, so at the quark level their decays are caused by $c \rightarrow s / d$ and $b \rightarrow u / c$ transitions. A typical diagram of such processes is shown in Fig. 1. We should note that there exists also an unfactorized case, when the light quark from a baryon takes part in the production of $\pi$. However, the contribution of such a diagram is small due to color suppression and its calculation is not a subject of this paper.

According to the factorization theorem process, $\mathcal{B}_{1} \rightarrow$ $\mathcal{B}_{2} R$ can be separated into two independent parts: weak decay $\mathcal{B}_{1} \rightarrow \mathcal{B}_{2} W$ and the $W \rightarrow R$ transition. As a result its matrix element can be written as

$$
\mathcal{M}=\frac{G_{F}}{\sqrt{2}} V_{i j} a_{1} H^{\mu} \varepsilon_{\mu},
$$



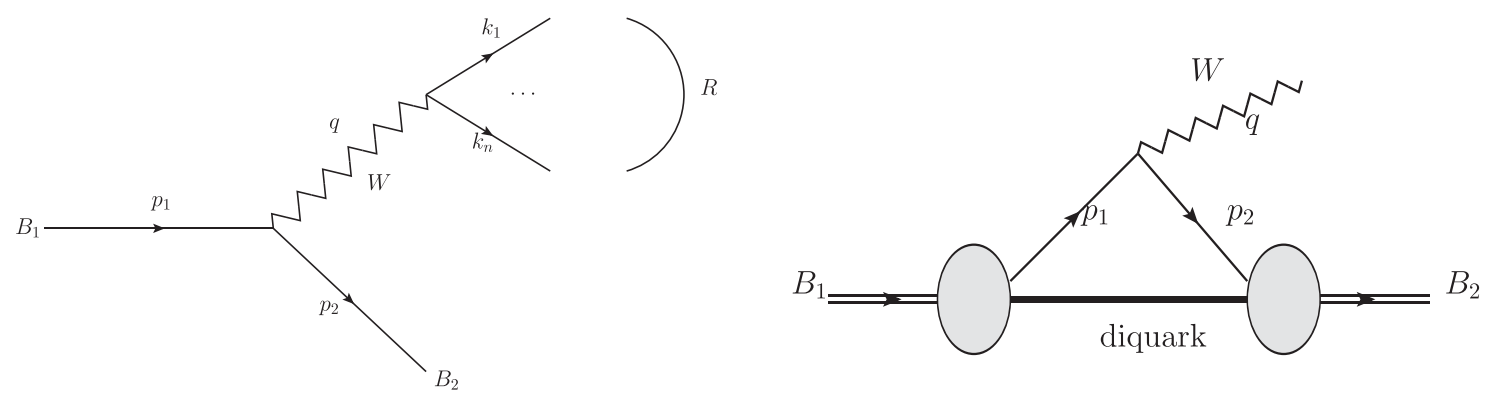

FIG. 1. Typical diagrams for weak decays $\mathcal{B}_{1} \rightarrow \mathcal{B}_{2} R$.

where $G_{F}$ is the Fermi constant, $V_{i j}$ the CabibboKobayashi-Maskawa (CKM) matrix element, constant $a_{1}$ describes soft gluon rescattering [3], $\varepsilon_{\mu}$ is the effective polarization vector of the $W$-boson, and the amplitude of the $\mathcal{B}_{1} \rightarrow \mathcal{B}_{2}$ transition is denoted as $H^{\mu}$.

\section{A. $\mathcal{B}_{1} \rightarrow \mathcal{B}_{2} W$ transition}

This amplitude can be written as [4]

$$
\begin{aligned}
H^{\mu}= & \bar{u}\left(P^{\prime}\right)\left[\gamma^{\mu} f_{1}\left(q^{2}\right)+i \sigma^{\mu \nu} \frac{q_{\nu}}{M_{1}} f_{2}\left(q^{2}\right)+\frac{q^{\mu}}{M_{1}} f_{3}\left(q^{2}\right)\right] u(P) \\
& -\bar{u}\left(P^{\prime}\right)\left[\gamma^{\mu} g_{1}\left(q^{2}\right)+i \sigma^{\mu \nu} \frac{q_{\nu}}{M_{1}} g_{2}\left(q^{2}\right)+\frac{q^{\mu}}{M_{1}} g_{3}\left(q^{2}\right)\right] \\
& \times \gamma_{5} u(P)
\end{aligned}
$$

where

$$
\sigma^{\mu \nu}=i\left(\gamma^{\mu} \gamma^{\nu}-\gamma^{\nu} \gamma^{\mu}\right) / 2,
$$

$M_{1}$ is the mass of the initial baryon, $P\left(P^{\prime}\right)$ is the momentum of initial (final) baryon, $q=P^{\prime}-P$ is the transferred momentum, and $f_{i}\left(q^{2}\right)$ and $g_{i}\left(q^{2}\right)$ are the form factors of the $\mathcal{B}_{1} \rightarrow \mathcal{B}_{2}$ transition. The contributions of the $f_{3}$ and $g_{3}$ form factors are suppressed as $\left(m_{u}+m_{d}\right) / m_{\mathcal{B}}$ and we will neglect them in the following.

In the calculation of form factors we use the quarkdiquark model, in which the baryon is considered to be built from scalar or axial diquarks (with spin $S_{d}=0$ and 1, respectively) and a single quark. It is convenient to take those quarks as diquarks that do not take part in weak decay. It is clear that in this approximation spin and the internal state of the diquark remain the same and, for example, in the case of $\Omega_{b c}^{0} \rightarrow \Omega_{c c}^{+} W^{-}$decay it is required to consider form factors of the

$$
b(c s)_{S, A} \rightarrow c(c s)_{S, A}+W^{-}
$$

transitions. In the above expression, indexes $S$ and $A$ correspond to the scalar and axial diquark. As a result, the form factor of this decay is equal to

$$
F\left(q^{2}\right)=c_{S} F_{S}\left(q^{2}\right)+c_{A} F_{A}\left(q^{2}\right),
$$

where $F_{S, A}\left(q^{2}\right)$ and $c_{S, A}$ are the form factors and coefficients of scalar (axial) diquarks, respectively. The form factors can be calculated in the framework of the potential model and, according to [4], are determined by overlap integrals. A detailed presentation of the calculation of the form factors can be found in $[4,5]$. As for the coefficients $c_{S, A}$, their values can be determined using Clebsch-Gordan coefficients.

Let us consider baryon $\Omega_{c c}^{+}$as an example. In this case, the diquark $c c$ can only have spin $S_{d}=1$ (the scalar diquark option is forbidden by the Pauli exclusion principle), so its spinor wave function can be written as

$$
(c c)_{A}=c_{1}(\uparrow) c_{2}(\uparrow)
$$

where symbols $\uparrow$ and $\downarrow$ mark states of the $c$-quark with spin projections $S_{z}=1 / 2$ and $-1 / 2$, respectively. The wave function of the baryon with full spin $S=1 / 2$ can be written in the same way:

$$
\Omega_{c c}^{+}=c_{1}(\uparrow) c_{2}(\uparrow) s(\downarrow) .
$$

In the $c(c s)$ basis, on the other hand, this wave function changes to

$$
\begin{aligned}
\Omega_{c c}^{+}= & \frac{1}{\sqrt{2}}\left[-\frac{\sqrt{3}}{2} c_{1}(\uparrow)\left(c_{2} s\right)_{S}\right. \\
& \left.+\frac{1}{2} c_{1}(\downarrow)\left(c_{2} s\right)_{A}+\left(c_{1} \leftrightarrow c_{2}\right)\right] .
\end{aligned}
$$

For the baryon $\Omega_{b c}^{0}$, the wave function is

$$
\Omega_{b c}^{0}=-\frac{\sqrt{3}}{2} b(\uparrow)(c s)_{S}+\frac{1}{2} b(\downarrow)(c s)_{A} .
$$

From these expressions for decay $\Omega_{b c}^{0} \rightarrow \Omega_{c c}^{+} R$ we get

$$
c_{S}=\frac{3 \sqrt{2}}{4}, \quad c_{A}=\frac{\sqrt{2}}{4} .
$$

In the Appendix we show the values of these coefficients and parametrization of the form factors for all of the decays considered in our paper. 


\section{B. Spectral function formalism}

The width of the $\mathcal{B}_{1} \rightarrow \mathcal{B}_{2} R$ decay is equal to

$$
\Gamma=\frac{1}{2} \frac{1}{2 M_{1}} \int d \Phi_{n+1}|\mathcal{M}|^{2},
$$

where the Lorentz-invariant phase space $d \Phi_{n}$ is defined by the expression

$$
d \Phi_{n}\left(q \rightarrow k_{1} \ldots k_{n}\right)=(2 \pi)^{4} \delta^{4}\left(q-\sum k_{i}\right) \prod \frac{d^{3} k_{i}}{(2 \pi)^{3} 2 E_{i}} .
$$

The following recurrence relation holds for this phase space:

$$
\begin{aligned}
d \Phi_{n+1}\left(p_{1} \rightarrow p_{2} k_{1} \ldots k_{n}\right)= & \frac{d q^{2}}{2 \pi} d \Phi_{2}\left(p_{1} \rightarrow p_{2} q\right) \\
& \times d \Phi_{n}\left(q \rightarrow k_{1} \ldots k_{n}\right) \\
= & d q^{2} \frac{\lambda}{8 \pi} \frac{d \Phi_{n}\left(q \rightarrow k_{1} \ldots k_{n}\right)}{2 \pi},
\end{aligned}
$$

where

$$
\lambda=\sqrt{1-\left(\frac{M_{2}+\sqrt{q^{2}}}{M_{1}}\right)^{2}} \sqrt{1-\left(\frac{M_{2}-\sqrt{q^{2}}}{M_{1}}\right)^{2}},
$$

and $M_{2}$ is the mass of the final baryon. Summed over polarizations, the squared matrix element (2) can be written in the following form:

$$
\sum|\mathcal{M}|^{2}=\frac{G_{F}^{2}}{2} V_{i j}^{2} a_{1}^{2} H^{\mu} H^{\nu *} \varepsilon_{\mu} \varepsilon_{\nu}^{*}
$$

The decay width distribution over the square transferred momentum with the help of these expressions can be written as

$$
\frac{d \Gamma}{d q^{2}}=\frac{G_{F}^{2}}{2} V_{i j}^{2} a_{1}^{2} \frac{1}{2 M_{1}} \frac{1}{2} \frac{\lambda}{8 \pi}\left(H_{T}^{2} \rho_{T}+H_{L}^{2} \rho_{L}\right),
$$

where $\rho_{L, T}\left(q^{2}\right)$ are (dependent on the final state $R$ ) longitudinal and transverse spectral functions, defined according to

$$
\begin{aligned}
\int & \frac{d \Phi_{n}\left(q \rightarrow k_{1} \ldots k_{n}\right)}{2 \pi} \varepsilon_{\mu} \varepsilon_{\nu}^{*} \\
& =\left(q_{\mu} q_{\nu}-q^{2} g_{\mu \nu}\right) \rho_{T}\left(q^{2}\right)+q_{\mu} q_{\nu} \rho_{L}\left(q^{2}\right),
\end{aligned}
$$

while squared longitudinal and transverse matrix elements are equal to

$$
\begin{aligned}
H_{T}^{2}= & H^{\mu} H^{\nu *}\left(q_{\mu} q_{\nu}-q^{2} g_{\mu \nu}\right) \\
= & \frac{1}{2 M_{1}^{2}}\left(f_{1}^{2} M_{1}^{2}\left(-2 q^{4}+2 q^{2} M_{-}^{2}-q^{2} M_{+}^{2}+M_{-}^{2} M_{+}^{2}\right)\right. \\
& +12 f_{1} f_{2} q^{2} M_{+}\left(q^{2}-M_{-}^{2}\right) M_{1}-4 f_{2}^{2} q^{2}\left(q^{4}-q^{2} M_{-}^{2}\right. \\
& \left.+2 q^{2} M_{+}^{2}-2 M_{-}^{2} M_{+}^{2}\right)+g_{1}^{2}\left(M_{-}+M_{+}\right)^{2} \\
& \times\left(-2 q^{4}-q^{2} M_{-}^{2}+2 q^{2} M_{+}^{2}+M_{-}^{2} M_{+}^{2}\right) \\
& +12 g_{1} g_{2} q^{2} M_{-}\left(M_{+}^{2}-q^{2}\right)\left(M_{-}+M_{+}\right) \\
& \left.-4 g_{2}^{2} q^{2}\left(q^{4}+2 q^{2} M_{-}^{2}-q^{2} M_{+}^{2}-2 M_{-}^{2} M_{+}^{2}\right)\right) \\
H_{L}^{2}= & H^{\mu} H^{\nu *} q_{\mu} q_{\nu} \\
= & 2\left(f_{1}^{2} M_{-}^{2}\left(M_{+}^{2}-\mathrm{q}^{2}\right)+g_{1}^{2} M_{+}^{2}\left(M_{-}-\mathrm{q}^{2}\right)\right),
\end{aligned}
$$

where $M_{ \pm}=M_{1} \pm M_{2}$.

\section{CALCULATIONS OF SPECTRAL FUNCTIONS}

In several cases the spectral functions can be easily calculated. For example, for semileptonic decay (see its diagram in Fig. 2), the effective polarization vector of the $W$-boson is equal to

$$
\varepsilon_{\mu}=\bar{u}_{\nu_{l}}(k) \gamma_{\mu}\left(1+\gamma_{5}\right) u_{l}(p),
$$

where $p$ and $k$ are the momenta of final leptons (we assume that their masses are negligible). It is easy to show that summed over leptons' polarization, the square of this vector is equal to

$\sum_{\text {pol }} \varepsilon_{\mu} \varepsilon_{\nu}^{*}=8\left(-g_{\mu \nu}(p k)+k_{\mu} p_{\nu}+k_{\nu} p_{\mu}+i e_{\mu \nu \alpha \beta} p^{\alpha} k^{\beta}\right)$.

As a result, in this case the longitudinal spectral function is identically equal to zero (we could expect this because of the partial conservation of the axial current). For the transversal one we have

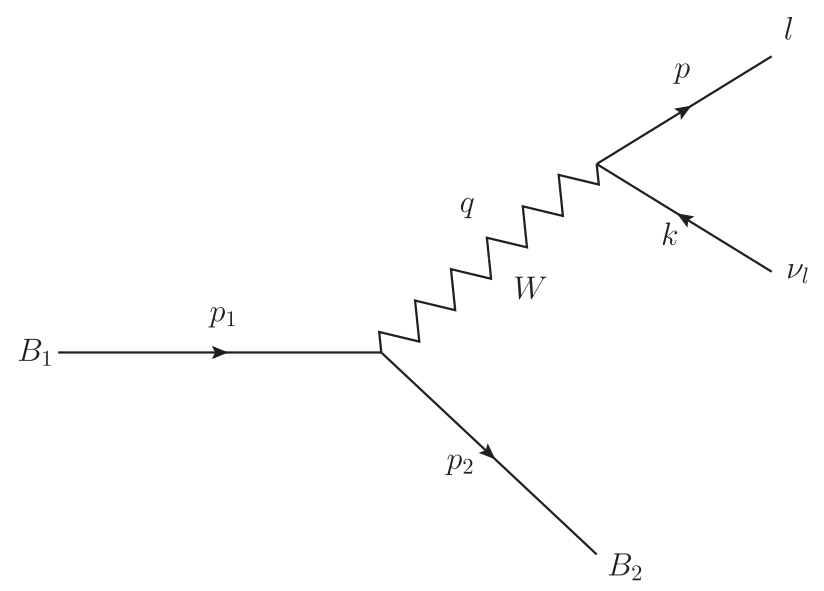

FIG. 2. Diagram for semileptonic decay. 


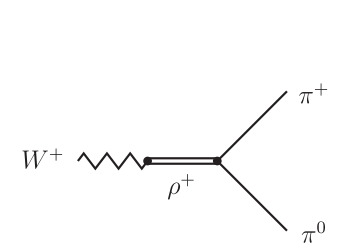

(a)

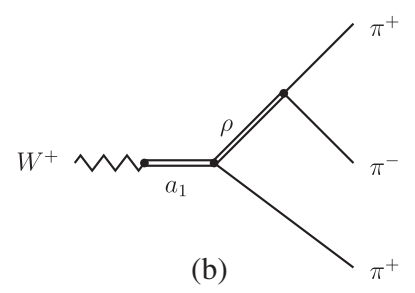

(b)

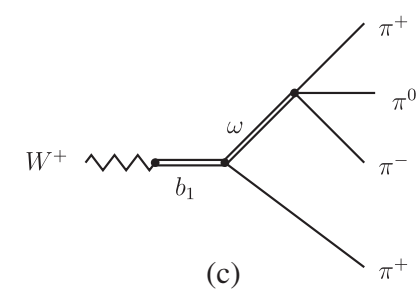

(c)

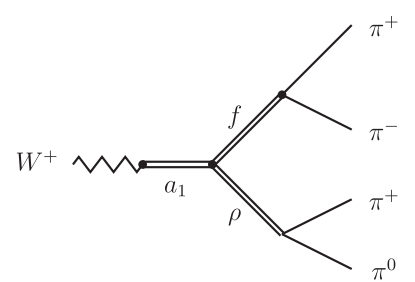

(d)

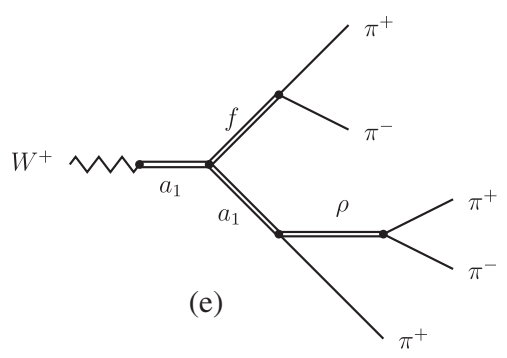

FIG. 3. Diagrams for transitions $W \rightarrow 2 \pi, W \rightarrow 3 \pi, W \rightarrow 4 \pi$, and $W \rightarrow 5 \pi$.

$$
\rho_{T}^{\left(l \nu_{l}\right)}\left(q^{2}\right)=\frac{1}{6 \pi^{2}} .
$$

Diagrams for decays to $\pi$ and $\rho$ mesons are trivial. It is well known that amplitudes of the $W \rightarrow \pi$ and $W \rightarrow \rho$ transitions are defined as

$$
\left\langle\pi\left|J_{\mu}\right| W\right\rangle=f_{\pi} k_{\mu}, \quad\left\langle\rho\left|J_{\mu}\right| W\right\rangle=f_{\rho} m_{\rho} \varepsilon_{\mu}^{*},
$$

respectively, where $f_{\pi}=130 \mathrm{MeV}, f_{\rho}=216 \mathrm{MeV}$, and $m_{\rho}=775 \mathrm{MeV}$ [6]. As a result we have

$$
\begin{aligned}
& \rho_{T}^{(\pi)}\left(q^{2}\right)=0, \quad \rho_{L}^{(\pi)}=f_{\pi}^{2} \delta\left(q^{2}-m_{\pi}^{2}\right), \\
& \rho_{T}^{(\rho)}\left(q^{2}\right)=f_{\rho}^{2} \delta\left(q^{2}-m_{\rho}^{2}\right), \quad \rho_{L}^{(\rho)}=0 .
\end{aligned}
$$

For the $W \rightarrow 3 \pi, 4 \pi, 5 \pi$ transitions, the longitudinal spectral functions are equal to zero because of the partial conservation of the axial current. The shapes of the transversal functions, on the other hand, can be determined using the resonance model (see Fig. 3). The normalization of these functions can be found using experimental values of the branching fractions of the $\tau \rightarrow \nu_{\tau}+R$ decays [6-8]. For these processes

$$
\begin{gathered}
\left.H^{\mu}=\bar{u}\left(P_{\nu_{\tau}}\right) \gamma^{\mu}\left(1+\gamma_{5}\right) u\right)\left(P_{\tau}\right) \\
\Gamma\left(\tau \rightarrow \nu_{\tau} R\right)=\frac{G_{F}^{2}}{2} \frac{1}{2 m_{\tau}} \frac{\lambda}{8 \pi} \int d q^{2}\left(m_{\tau}^{4}+m_{\tau}^{2} q^{2}-2 q^{4}\right) \rho_{T},
\end{gathered}
$$

where $m_{\tau}=1776 \mathrm{MeV}$ [6] is the mass of the $\tau$ lepton, $P_{\tau}$ is its momentum, and $P_{\nu_{\tau}}$ is the momentum of the $\tau$-neutrino. Analytical calculations of the corresponding spectral functions are rather complicated, so we made a numerical integration using created by our group EvtGen [9] models [10-13].

In Fig. 4 we show the $q^{2}$ dependence of these spectral functions. It can be easily seen that the $\rho_{T}^{2 \pi}$ spectral function has a clear peak in the $q^{2} \approx 0.5 \mathrm{GeV}^{2}$ region and a little bump at somewhat higher energy. These peaks correspond to contributions of the virtual $\rho$ meson and its excitations. As for $\rho_{T}^{(3 \pi)}$, this spectral function has a peak in the $q^{2} \approx 1 \mathrm{GeV}^{2}$ region. This peak corresponds to the virtual $a_{1}$ meson shown in Fig. 3(b), whose mass is $m_{a_{1}} \approx 1.2 \mathrm{GeV}$ [6]. The same meson also produces peaks in $\rho_{T}^{(4 \pi)}$ and $\rho_{T}^{(5 \pi)}$ spectral functions. These peaks, however, are shifted to higher energy region by multibody phase space. It is interesting to note also that in the case of $4 \pi$ production both $a_{1}$ and $b_{1}$ mesons can contribute [see Figs. 3(c) and (d)]. Theoretically we should consider the interference between these two channels, but, since in the latter case three pions are produced in the decay of the narrow $\omega$ meson, such interference can be neglected.

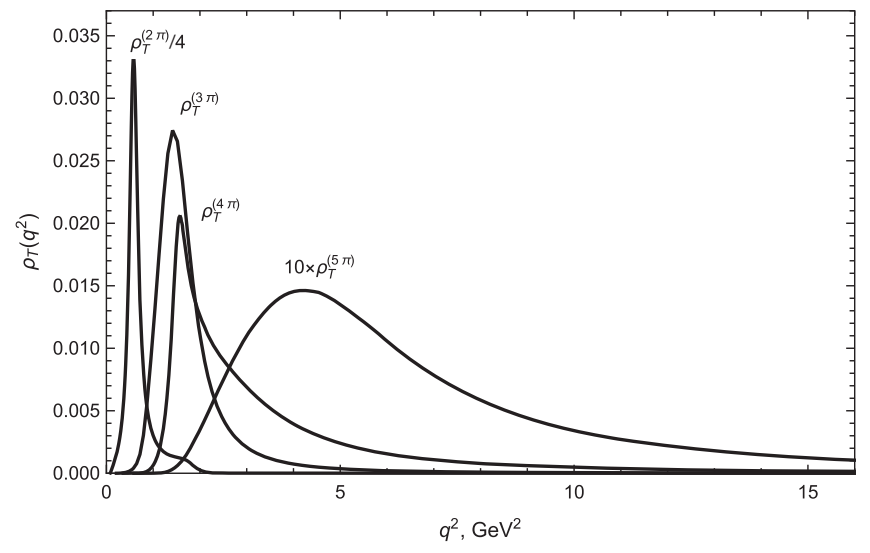

FIG. 4. Spectral functions. 
TABLE I. Masses (in $\mathrm{GeV}$ ) and decay widths (in $\mathrm{ps}^{-1}$ ) of initial and final doubly heavy baryons.

\begin{tabular}{|c|c|c|c|c|c|c|c|c|c|c|c|}
\hline $\mathcal{B}$ & $m_{\mathcal{B}}$ & $\Gamma_{\mathcal{B}}$ & $\mathcal{B}$ & $m_{\mathcal{B}}$ & $\Gamma_{\mathcal{B}}$ & $\mathcal{B}$ & $m_{\mathcal{B}}$ & $\mathcal{B}$ & $m_{\mathcal{B}}$ & $\mathcal{B}$ & $m_{\mathcal{B}}$ \\
\hline$\Xi_{c c}^{++}$ & 3.627 & 3.3 & $\Xi_{c c}^{+}$ & 3.627 & 10.0 & $\Lambda_{c}^{+}$ & 2.286 & $\Sigma_{c}^{++}$ & 2.454 & $\Sigma_{c}^{+}$ & 2.453 \\
\hline$\Omega_{c c}^{+}$ & 3.65 & 3.7 & $\Xi_{b b}^{0}$ & 10.31 & 2.7 & $\Sigma_{c}^{0}$ & 2.454 & $\Xi_{c}^{+}$ & 2.486 & $\Xi_{c}^{\prime+}$ & 2.576 \\
\hline$\Xi_{b b}^{-}$ & 10.32 & 2.7 & $\Omega_{b b}^{-}$ & 10.45 & 1.2 & $\Xi_{c}^{0}$ & 2.471 & $\Xi_{c}^{\prime 0}$ & 2.578 & $\Omega_{c}^{0}$ & 2.695 \\
\hline$\Xi_{b c}^{+}$ & 6.914 & 4.1 & $\Xi_{b c}^{0}$ & 6.914 & 11.0 & $\Lambda_{b}^{0}$ & 5.620 & $\Sigma_{b}^{+}$ & 5.811 & $\Sigma_{b}^{0}$ & 5.814 \\
\hline$\Omega_{b c}^{0}$ & 7.136 & 4.5 & $\Xi_{b c}^{D c}$ & 6.914 & 4.1 & $\Sigma_{b}^{-}$ & 5.816 & $\Xi_{b}^{0}$ & 5.793 & $\Xi_{b}^{\prime 0}$ & 5.935 \\
\hline$\Xi_{b c}^{\prime 0}$ & 6.914 & 11.0 & $\Omega_{b c}^{\prime 0}$ & 7.136 & 4.5 & $\Xi_{b}^{-}$ & 5.795 & $\Xi_{b}^{o}$ & 5.935 & $\Omega_{b}^{-}$ & 6.046 \\
\hline
\end{tabular}

Comparison with later experimental results [14-17] showed that these spectral functions are well suited for theoretical description of exclusive decays of $B_{c}$-mesons, so we can expect that for the processes in the case studied in our paper they can also be used.

\section{NUMERICAL RESULTS}

For numerical calculation we use values for the Fermi constant and CKM matrix elements given in PDG [6]:
$G_{F}=1.166 \times 10^{-5} \mathrm{GeV}^{-2}$,

$V_{u d}=0.974, \quad V_{u s}=0.225, \quad V_{u b}=0.00357$,

$V_{c d}=0.225, \quad V_{c s}=0.974, \quad V_{c b}=0.0411$.

Values of masses of initial and final baryons are presented in Table I.

Using the expressions presented in the previous section, it is easy to calculate the branching fractions of the decays

TABLE II. Branching fractions of $c c$ baryons in percents.

\begin{tabular}{|c|c|c|c|c|c|c|c|c|}
\hline \multirow[b]{2}{*}{$\mathcal{B}_{1} \rightarrow \mathcal{B}_{2}$} & \multicolumn{8}{|c|}{ Modes } \\
\hline & $l \nu_{l}$ & $\pi$ & $\rho$ & $2 \pi$ & $3 \pi$ & $4 \pi($ ex. $\omega)$ & $4 \pi$ & $5 \pi$ \\
\hline$\Xi_{c c}^{++} \rightarrow \Lambda_{c}^{+}$ & 0.494 & 0.377 & 0.993 & 0.863 & 0.208 & 0.0302 & 0.0637 & $2.2 \times 10^{-4}$ \\
\hline$\Xi_{c c}^{++} \rightarrow \Sigma_{c}^{+}$ & 0.45 & 0.302 & 1.05 & 0.9 & 0.154 & 0.0101 & 0.0189 & $4.0 \times 10^{-5}$ \\
\hline$\Xi_{c c}^{++} \rightarrow \Xi_{c}^{+}$ & 4.99 & 8.3 & 12.3 & 10.0 & 0.634 & 0.0253 & 0.046 & $6.0 \times 10^{-5}$ \\
\hline$\Xi_{c c}^{++} \rightarrow \Xi_{c}^{\prime+}$ & 5.98 & 6.93 & 17.6 & 14.1 & 0.725 & 0.0172 & 0.0307 & $3.0 \times 10^{-5}$ \\
\hline$\Xi_{c c}^{+} \rightarrow \Sigma_{c}^{0}$ & 0.299 & 0.201 & 0.698 & 0.598 & 0.101 & 0.00659 & 0.0124 & $3.0 \times 10^{-5}$ \\
\hline$\Xi_{c c}^{+} \rightarrow \Xi_{c}^{0}$ & 1.65 & 2.75 & 4.08 & 3.33 & 0.21 & 0.00837 & 0.0152 & $2.0 \times 10^{-5}$ \\
\hline$\Xi_{c c}^{+} \rightarrow \Xi_{c}^{\prime 0}$ & 1.98 & 2.31 & 5.86 & 4.68 & 0.238 & 0.0056 & 0.00999 & $1.0 \times 10^{-5}$ \\
\hline$\Omega_{c c}^{+} \rightarrow \Xi_{c}^{0}$ & 0.208 & 0.293 & 0.512 & 0.421 & 0.0342 & 0.00166 & 0.00306 & $1.0 \times 10^{-5}$ \\
\hline$\Omega_{c c}^{+} \rightarrow \Xi_{c}^{\prime 0}$ & 0.249 & 0.244 & 0.711 & 0.577 & 0.039 & 0.0011 & 0.00199 & $1.0 \times 10^{-5}$ \\
\hline$\Omega_{c c}^{+} \rightarrow \Omega_{c}^{0}$ & 6.66 & 11.2 & 22.6 & 16.9 & 0.265 & 0.00357 & 0.00479 & $1.0 \times 10^{-5}$ \\
\hline
\end{tabular}

TABLE III. Branching fractions of $b c$ baryons in percents.

\begin{tabular}{|c|c|c|c|c|c|c|c|c|}
\hline \multirow[b]{2}{*}{$\mathcal{B}_{1} \rightarrow \mathcal{B}_{2}$} & \multicolumn{8}{|c|}{ Modes } \\
\hline & $l \nu_{l}$ & $\pi$ & $\rho$ & $2 \pi$ & $3 \pi$ & $4 \pi($ ex. $\omega)$ & $4 \pi$ & $5 \pi$ \\
\hline$\overline{\Xi_{b c}^{+}} \rightarrow \Lambda_{b}^{0}$ & 0.223 & 0.0438 & 0.486 & 0.415 & 0.0781 & 0.00879 & 0.0187 & $5 . \times 10^{-5}$ \\
\hline$\Xi_{b c}^{+} \rightarrow \Sigma_{b}^{0}$ & 0.148 & 0.0306 & 0.403 & 0.332 & 0.0292 & 0.00103 & 0.00188 & 1. $\times 10^{-5}$ \\
\hline$\Xi_{b c}^{+} \rightarrow \Xi_{b}^{0}$ & 2.3 & 0.927 & 5.84 & 4.72 & 0.248 & 0.00837 & 0.0151 & 2. $\times 10^{-5}$ \\
\hline$\Xi_{b c}^{00} \rightarrow \Sigma_{b}^{-}$ & 0.112 & 0.0233 & 0.306 & 0.251 & 0.0217 & $7.5 \times 10^{-4}$ & 0.00137 & 1. $\times 10^{-5}$ \\
\hline$\Xi_{b c}^{0} \rightarrow \Xi_{b}^{-}$ & 0.868 & 0.353 & 2.21 & 1.78 & 0.0922 & 0.00306 & 0.00552 & 1. $\times 10^{-5}$ \\
\hline$\Omega_{b c}^{0} \rightarrow \Xi_{b}^{-}$ & 0.254 & 0.0384 & 0.511 & 0.443 & 0.105 & 0.0148 & 0.0313 & $1.1 \times 10^{-4}$ \\
\hline$\Omega_{b c}^{0} \rightarrow \Omega_{b}^{-}$ & 6.03 & 1.25 & 16.6 & 13.6 & 1.07 & 0.0344 & 0.0628 & 7. $\times 10^{-5}$ \\
\hline$\Xi_{b c}^{+} \rightarrow \Sigma_{c}^{++}$ & 0.0035 & $2.63 \times 10^{-6}$ & $2.37 \times 10^{-4}$ & $2.4 \times 10^{-4}$ & $2.1 \times 10^{-4}$ & $2.7 \times 10^{-4}$ & $3.3 \times 10^{-4}$ & 0.0016 \\
\hline$\Xi_{b c}^{+} \rightarrow \Xi_{c c}^{++}$ & 1.58 & 0.00803 & 0.438 & 0.414 & 0.289 & 0.268 & 0.341 & 0.0703 \\
\hline$\Xi_{b c}^{0} \rightarrow \Lambda_{c}^{+}$ & $3.0 \times 10^{-4}$ & $5.1 \times 10^{-7}$ & $4.3 \times 10^{-5}$ & $5.0 \times 10^{-5}$ & $4.0 \times 10^{-5}$ & $4.0 \times 10^{-5}$ & $4.0 \times 10^{-5}$ & $1.6 \times 10^{-4}$ \\
\hline$\Xi_{b c}^{b c} \rightarrow \Sigma_{c}^{+}$ & $7.0 \times 10^{-4}$ & $5.0 \times 10^{-7}$ & $4.6 \times 10^{-5}$ & $5.0 \times 10^{-5}$ & $4.0 \times 10^{-5}$ & $6.0 \times 10^{-5}$ & $7.0 \times 10^{-5}$ & $3.1 \times 10^{-4}$ \\
\hline$\Xi_{b c}^{0} \rightarrow \Xi_{c c}^{+}$ & 0.603 & 0.00305 & 0.167 & 0.157 & 0.11 & 0.102 & 0.13 & 0.0267 \\
\hline$\Omega_{b c}^{0} \rightarrow \Xi_{c}^{+}$ & $5.0 \times 10^{-4}$ & $9.4 \times 10^{-7}$ & $8.8 \times 10^{-5}$ & $9.0 \times 10^{-5}$ & $7.0 \times 10^{-5}$ & $7.0 \times 10^{-5}$ & $9.0 \times 10^{-5}$ & $2.6 \times 10^{-4}$ \\
\hline$\Omega_{b c}^{0} \rightarrow \Omega_{c c}^{+}$ & 1.87 & 0.00663 & 0.429 & 0.407 & 0.289 & 0.279 & 0.353 & 0.0784 \\
\hline
\end{tabular}


TABLE IV. Branching fractions of $b b$ baryons in percents.

\begin{tabular}{|c|c|c|c|c|c|c|c|c|}
\hline \multirow[b]{2}{*}{$\mathcal{B}_{1} \rightarrow \mathcal{B}_{2}$} & \multicolumn{8}{|c|}{ Modes } \\
\hline & $l \nu_{l}$ & $\pi$ & $\rho$ & $2 \pi$ & $3 \pi$ & $4 \pi($ ex. $\omega)$ & $4 \pi$ & $5 \pi$ \\
\hline$\Xi_{b b}^{0} \rightarrow \Sigma_{b}^{+}$ & 0.0043 & $1.43 \times 10^{-6}$ & $4.34 \times 10^{-4}$ & $4.3 \times 10^{-4}$ & $3.8 \times 10^{-4}$ & $4.7 \times 10^{-4}$ & $5.8 \times 10^{-4}$ & 0.00128 \\
\hline$\Xi_{b b}^{0} \rightarrow \Xi_{b c}^{+}$ & 2.59 & 0.00347 & 0.568 & 0.54 & 0.399 & 0.397 & 0.499 & 0.113 \\
\hline$\Xi_{b b}^{0} \rightarrow \Xi_{b c}^{\prime+}$ & 1.15 & $6.26 \times 10^{-4}$ & 0.128 & 0.125 & 0.113 & 0.141 & 0.172 & 0.0499 \\
\hline$\Xi_{b b}^{-} \rightarrow \Lambda_{b}^{0}$ & 0.0011 & $8.3 \times 10^{-7}$ & $2.23 \times 10^{-4}$ & $2.2 \times 10^{-4}$ & $1.7 \times 10^{-4}$ & $1.6 \times 10^{-4}$ & $2.1 \times 10^{-4}$ & $3.1 \times 10^{-4}$ \\
\hline$\Xi_{b b}^{-} \rightarrow \Sigma_{b}^{0}$ & 0.0022 & $7.2 \times 10^{-7}$ & $2.18 \times 10^{-4}$ & $2.2 \times 10^{-4}$ & $1.9 \times 10^{-4}$ & $2.4 \times 10^{-4}$ & $2.9 \times 10^{-4}$ & $6.5 \times 10^{-4}$ \\
\hline$\Xi_{b b}^{-} \rightarrow \Xi_{b c}^{0}$ & 2.62 & 0.00349 & 0.572 & 0.544 & 0.402 & 0.4 & 0.504 & 0.115 \\
\hline$\Xi_{b b}^{-} \rightarrow \Xi_{b c}^{\prime c}$ & 1.16 & $6.23 \times 10^{-4}$ & 0.128 & 0.125 & 0.113 & 0.142 & 0.172 & 0.05 \\
\hline$\Omega_{b b}^{-} \rightarrow \Xi_{b}^{0}$ & 0.002 & $1.64 \times 10^{-6}$ & $4.51 \times 10^{-4}$ & $4.4 \times 10^{-4}$ & $3.3 \times 10^{-4}$ & $3.2 \times 10^{-4}$ & $4.1 \times 10^{-4}$ & $4.7 \times 10^{-4}$ \\
\hline$\Omega_{b b}^{-} \rightarrow \Xi_{b}^{\prime 0}$ & 0.0044 & $1.44 \times 10^{-6}$ & $4.53 \times 10^{-4}$ & $4.5 \times 10^{-4}$ & 4. $\times 10^{-4}$ & $4.9 \times 10^{-4}$ & 6. $\times 10^{-4}$ & 0.00134 \\
\hline$\Omega_{b b}^{-} \rightarrow \Omega_{b c}^{0}$ & 4.81 & 0.00702 & 1.13 & 1.08 & 0.792 & 0.776 & 0.979 & 0.216 \\
\hline$\Omega_{b b}^{-} \rightarrow \Omega_{b c}^{\prime 0}$ & 2.13 & 0.00126 & 0.256 & 0.251 & 0.226 & 0.28 & 0.342 & 0.0966 \\
\hline
\end{tabular}

considered in our paper (see Tables II-IV). We can observe a perfect agreement with presented in paper [4] results in the case $B_{1} \rightarrow B_{2} \mu \nu, B_{1} \rightarrow B_{2} \pi$, and $B_{1} \rightarrow B_{2} \rho$ decays. It should be stressed, however, that in the case of the latter final state the width of the $\rho$ meson is completely neglected in this work. It is clear, however, that in some cases such approximation could be unreliable. As a result, the branching fractions obtained in the framework of spectral function formalism (labeled $2 \pi$ in Tables II, III, and IV) differ from the results with the $\rho$-meson width neglected (see the

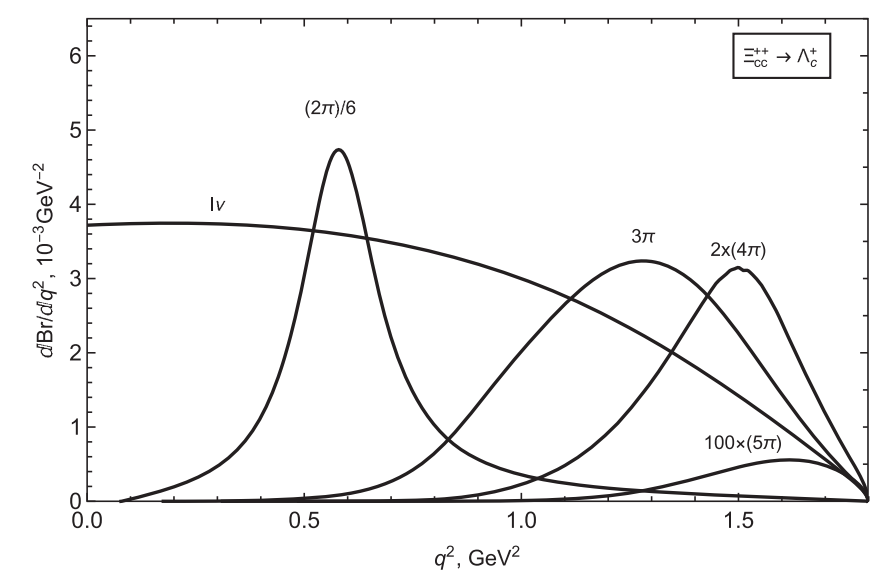

columns labeled $\rho$ in these tables). It should be noted also that depending on the initial and final baryons, the excitations of the $\rho$ meson increase the branching fraction of the $\mathcal{B}_{1} \rightarrow \mathcal{B}_{2} 2 \pi$ decays by approximately $1 \div 10 \%$.

The branching fractions of $3 \pi, 4 \pi$, and $5 \pi$ production are given in the last three columns of these tables. One can easily see that typically these processes are suppressed in comparison, for example, with single $\pi$ in the final state. It should be noted, however, that in the case of $b$ quark decay (see $\Xi_{b c}^{+} \rightarrow \Xi_{c c}^{++}$, for example) such suppression is not

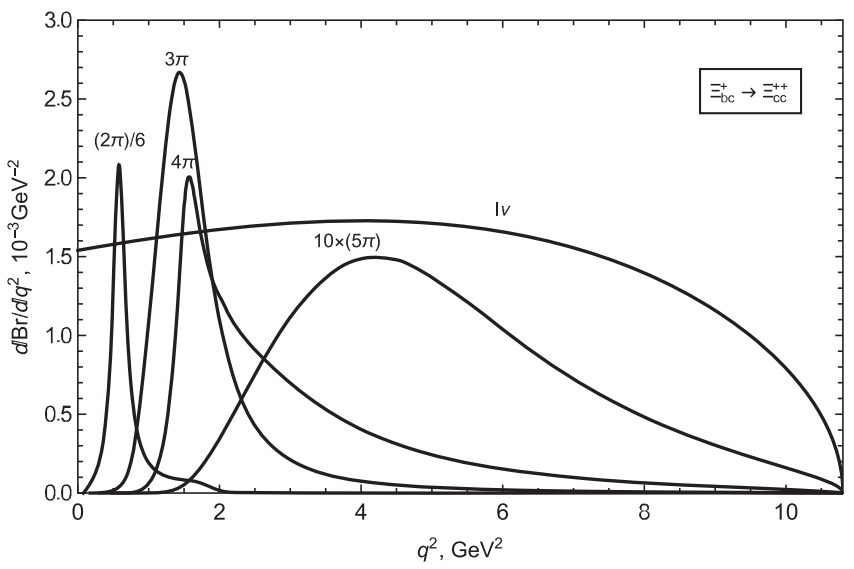

FIG. 5. $q^{2}$ distributions of the $\Xi_{c c}^{++} \rightarrow \Lambda_{c}^{+}$and $\Xi_{b c}^{+} \rightarrow \Xi_{c c}^{++} R$ branching fractions (left and right, respectively) for $R=\ell \nu, 2 \pi, 3 \pi, 4 \pi$, $5 \pi$. Note that some curves are scaled as shown in the figures.
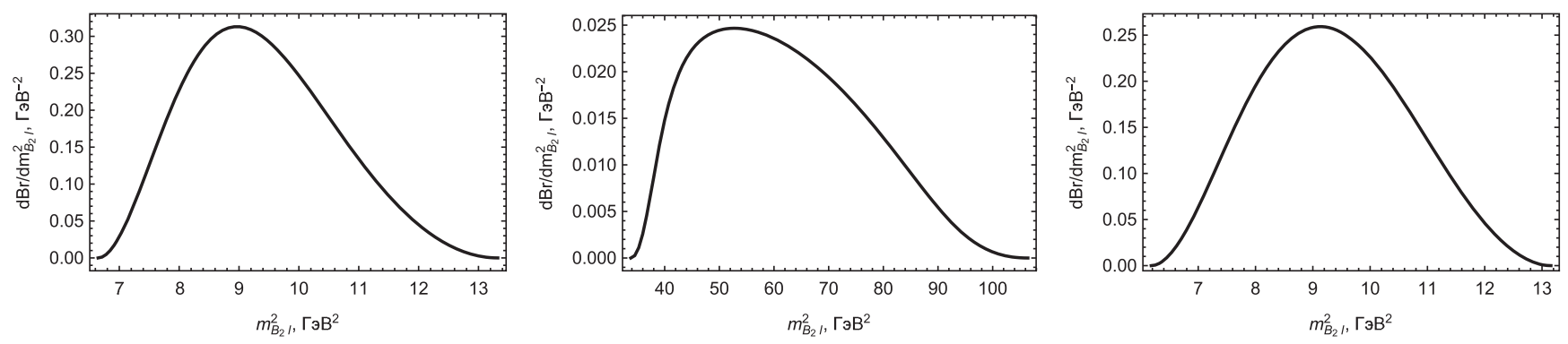

FIG. 6. $d \mathrm{Br} / d m_{\mathcal{B}_{2} l}^{2}$ for decays $\Omega_{c c}^{+} \rightarrow \Xi_{c}^{\prime 0} l \nu_{l}, \Xi_{b b}^{-} \rightarrow \Sigma_{b}^{0} l \nu_{l}, \Xi_{c c}^{+} \rightarrow \Xi_{c}^{+} l \nu_{l}$. 
observed. The reason is that in this case the energy deposit is large enough, so higher $q^{2}$ values are reachable. This is exactly the region where, according to Fig. 4, spectral functions of $W \rightarrow 4 \pi, 5 \pi$ transitions are large. In the case of c-quark decay, on the other hand, only the low $q^{2}$ region is available kinematically and in this region spectral functions of multiple quark production are small. This effect can be clearly seen in Fig. 5, where all $q^{2}$ distributions are shown for both the $\Xi_{c c}^{++} \rightarrow \Lambda_{c}^{+}$and $\Xi_{b c}^{+} \rightarrow \Xi_{c c}^{++}$transitions.

As for distributions over other Dalitz variables, it is clear that they cannot be obtained in the framework of spectral function formalism. In the case of three-body semileptonic decay, however, we can use a well-known Dalitz relation to calculate it. In Fig. 6, for example, we show the distributions over the invariant mass of the baryon-lepton pair for the same decays.

\section{CONCLUSION}

In the presented paper we have considered exclusive decays of the ground states of doubly heavy baryons $\Xi_{Q_{1} Q_{2}}$, $\Omega_{Q_{1} Q_{2}}$ (where $Q_{1,2}=c, b$ ) with production of the leptonic pair or the system of charged $\pi$ mesons. According to the factorization theorem, the widths of these processes can be written as a convolution of semileptonic decays and spectral functions that are connected with a virtual $W$-boson transition into a final system of light particles. The first stage can be described in terms of form factors of the weak decay, which can be calculated, for example, in the frame of potential models. The spectral functions, on the other hand, can be calculated, for example, from an analysis of $\tau$ lepton decays.

In our paper we gave analytical expressions for distributions of decay widths over the squared transferred momentum. Using the known parametrization of form factors of doubly heavy baryons and spectral functions, we gave numerical predictions of these distributions and integrated branching fractions. According to our results, for some of the processes the branchings are quite large, which can lead to their experimental observation. It should be noted that semileptonic decays of doubly heavy baryons were considered also in a number of other works (see, for example, $[5,18,19])$. The results of these works have some differences with ours, which is caused by the difference in the form factors' parametrization. Thereby, we think it is extremely interesting to try to experimentally observe marked decays.

In the future we plan to continue our work in this area. For example, it would be interesting to study the production of light mesons in decays of excited $P$-wave states of doubly heavy baryons. In addition, we plan to create, based on the presented theoretical models, Monte Carlo generators that are required for comparison of theoretical predictions with forthcoming experimental results.

\section{ACKNOWLEDGMENTS}

The authors are grateful to A. K. Likhoded for productive discussions and help in the preparation of this paper.

\section{APPENDIX: PARAMETRIZATION OF FORM FACTORS}

As shown in Sec. II, using results [4] in the case of the $\mathcal{B}_{1} \rightarrow \mathcal{B}_{2} W$ transition, we can obtain the dependence of the form factors on the squared transferred momentum for scalar and axial diquarks. In this paper it is more convenient to parametrize not the form factors themselves, but their sum,

$$
F\left(q^{2}\right)=c_{S} F_{S}\left(q^{2}\right)+c_{A} F_{A}\left(q^{2}\right) .
$$

In our paper we use the following parametrization:

$$
F\left(q^{2}\right)=F(0)\left(1+\alpha q^{2}+\beta q^{4}+\gamma q^{6}\right),
$$

where parameters $F(0), \alpha, \beta$, and $\gamma$ for different initial and final states are given in Tables $\mathrm{V}-\mathrm{X}$.

\begin{tabular}{|c|c|c|c|c|c|c|c|c|}
\hline \multirow[b]{2}{*}{$\mathcal{B}_{1} \rightarrow \mathcal{B}_{2}$} & \multicolumn{4}{|c|}{$f_{1}$} & \multicolumn{4}{|c|}{$f_{2}$} \\
\hline & $F(0)$ & $\alpha\left(\mathrm{GeV}^{-2}\right)$ & $\beta\left(\mathrm{GeV}^{-4}\right)$ & $\gamma\left(\mathrm{GeV}^{-6}\right)$ & $F(0)$ & $\alpha\left(\mathrm{GeV}^{-2}\right)$ & $\beta\left(\mathrm{GeV}^{-4}\right)$ & $\gamma\left(\mathrm{GeV}^{-6}\right)$ \\
\hline$\Xi_{c c}^{++} \rightarrow \Lambda_{c}^{+}$ & 0.791 & 0.386 & 0.118 & 0.016 & -0.00794 & -0.481 & -0.405 & -0.2 \\
\hline$\Xi_{c c}^{++} \rightarrow \Sigma_{c}^{+}$ & -0.467 & 0.294 & 0.0331 & 0.0417 & 1.04 & 0.418 & 0.108 & 0.037 \\
\hline$\Xi_{c c}^{++} \rightarrow \Xi_{c}^{+}$ & 0.914 & 0.348 & 0.0818 & 0.0187 & 0.0116 & 1.31 & 0.513 & 0.15 \\
\hline$\Xi_{c c}^{++} \rightarrow \Xi_{c}^{\prime+}$ & -0.538 & 0.247 & 0.0384 & 0.0213 & 1.11 & 0.366 & 0.0863 & 0.0276 \\
\hline$\Xi_{c c}^{+} \rightarrow \Sigma_{c}^{0}$ & -0.661 & 0.294 & 0.0332 & 0.0416 & 1.47 & 0.418 & 0.108 & 0.037 \\
\hline$\Xi_{c c}^{+} \rightarrow \Xi_{c}^{0}$ & 0.914 & 0.348 & 0.0818 & 0.0187 & 0.0116 & 1.31 & 0.513 & 0.15 \\
\hline$\Xi_{c c}^{+} \rightarrow \Xi_{c}^{\prime 0}$ & -0.538 & 0.247 & 0.0384 & 0.0213 & 1.11 & 0.366 & 0.0863 & 0.0276 \\
\hline$\Omega_{c c}^{+} \rightarrow \Xi_{c}^{0}$ & -0.783 & 0.406 & 0.117 & 0.0191 & 0.0214 & 0.194 & -0.0127 & -0.0214 \\
\hline$\Omega_{c c}^{+} \rightarrow \Xi_{c}^{\prime 0}$ & -0.462 & 0.308 & 0.0495 & 0.0408 & 1.05 & 0.425 & 0.116 & 0.0334 \\
\hline$\Omega_{c c}^{+} \rightarrow \Omega_{c}^{0}$ & -0.754 & 0.263 & 0.047 & 0.0205 & 1.59 & 0.376 & 0.0926 & 0.0244 \\
\hline
\end{tabular}

TABLE V. Parameters of form factors $f_{1}$ and $f_{2}$ for $c c$ baryons. 
TABLE VI. Parameters of form factors $g_{1}$ and $g_{2}$ for $c c$ baryons.

\begin{tabular}{|c|c|c|c|c|c|c|c|c|}
\hline \multirow[b]{2}{*}{$\mathcal{B}_{1} \rightarrow \mathcal{B}_{2}$} & \multicolumn{4}{|c|}{$g_{1}$} & \multicolumn{4}{|c|}{$g_{2}$} \\
\hline & $F(0)$ & $\alpha\left(\mathrm{GeV}^{-2}\right)$ & $\beta\left(\mathrm{GeV}^{-4}\right)$ & $\gamma\left(\mathrm{GeV}^{-6}\right)$ & $F(0)$ & $\alpha\left(\mathrm{GeV}^{-2}\right)$ & $\beta\left(\mathrm{GeV}^{-4}\right)$ & $\gamma\left(\mathrm{GeV}^{-6}\right)$ \\
\hline$\Xi_{c c}^{++} \rightarrow \Lambda_{c}^{+}$ & 0.224 & 0.235 & 0.0386 & -0.0045 & -0.0482 & 0.845 & -1.14 & 0.295 \\
\hline$\Xi_{c c}^{++} \rightarrow \Sigma_{c}^{+}$ & -0.624 & 0.244 & 0.0378 & 0.00399 & 0.0447 & 1.61 & -1.68 & 0.321 \\
\hline$\Xi_{c c}^{++} \rightarrow \Xi_{c}^{+}$ & 0.258 & 0.208 & 0.0262 & $-2.8 \times 10^{-4}$ & -0.0608 & 0.364 & 0.289 & -0.16 \\
\hline$\Xi_{c c}^{++} \rightarrow \Xi_{c}^{\prime+}$ & -0.728 & 0.216 & 0.0305 & 0.00386 & 0.0783 & 0.649 & 0.331 & -0.187 \\
\hline$\Xi_{c c}^{+} \rightarrow \Sigma_{c}^{0}$ & -0.883 & 0.244 & 0.0378 & 0.00399 & 0.0632 & 1.61 & -1.68 & 0.319 \\
\hline$\Xi_{c c}^{+} \rightarrow \Xi_{c}^{0}$ & 0.258 & 0.208 & 0.0262 & $-2.8 \times 10^{-4}$ & -0.0608 & 0.364 & 0.289 & -0.16 \\
\hline$\Xi_{c c}^{+} \rightarrow \Xi_{c}^{\prime 0}$ & -0.728 & 0.216 & 0.0305 & 0.00386 & 0.0783 & 0.649 & 0.33 & -0.187 \\
\hline$\Omega_{c c}^{+} \rightarrow \Xi_{c}^{0}$ & -0.222 & 0.249 & 0.0375 & -0.00273 & 0.0535 & 0.733 & -0.778 & 0.103 \\
\hline$\Omega_{c c}^{+} \rightarrow \Xi_{c}^{\prime 0}$ & -0.618 & 0.253 & 0.0398 & 0.00308 & 0.0511 & 1.3 & -0.874 & -0.133 \\
\hline$\Omega_{c c}^{+} \rightarrow \Omega_{c}^{0}$ & -1.02 & 0.225 & 0.0329 & 0.00381 & 0.119 & 0.671 & 0.297 & -0.159 \\
\hline
\end{tabular}

TABLE VII. Parameters of form factors $f_{1}$ and $f_{2}$ for $b c$ baryons.

\begin{tabular}{|c|c|c|c|c|c|c|c|c|}
\hline \multirow[b]{2}{*}{$\mathcal{B}_{1} \rightarrow \mathcal{B}_{2}$} & \multicolumn{4}{|c|}{$f_{1}$} & \multicolumn{4}{|c|}{$f_{2}$} \\
\hline & $F(0)$ & $\alpha\left(\mathrm{GeV}^{-2}\right)$ & $\beta\left(\mathrm{GeV}^{-4}\right)$ & $\gamma\left(\mathrm{GeV}^{-6}\right)$ & $F(0)$ & $\alpha\left(\mathrm{GeV}^{-2}\right)$ & $\beta\left(\mathrm{GeV}^{-4}\right)$ & $\gamma\left(\mathrm{GeV}^{-6}\right)$ \\
\hline$\Xi_{b c}^{+} \rightarrow \Lambda_{b}^{0}$ & 0.554 & 0.421 & 0.205 & -0.0498 & -0.297 & 0.425 & 0.211 & -0.05 \\
\hline$\Xi_{b c}^{+} \rightarrow \Sigma_{b}^{0}$ & -0.32 & 0.406 & 0.107 & 0.0123 & 1.54 & 0.453 & 0.155 & -0.0203 \\
\hline$\Xi_{b c}^{+} \rightarrow \Xi_{b}^{0}$ & 0.627 & 0.405 & 0.118 & -0.00891 & -0.301 & 0.423 & 0.131 & -0.0101 \\
\hline$\Xi_{b c}^{0} \rightarrow \Sigma_{b}^{-}$ & -0.453 & 0.407 & 0.107 & 0.0123 & 2.17 & 0.453 & 0.155 & -0.0201 \\
\hline$\Xi_{b c}^{0} \rightarrow \Xi_{b}^{-}$ & 0.627 & 0.405 & 0.118 & -0.00877 & -0.301 & 0.423 & 0.13 & -0.00994 \\
\hline$\Omega_{b c}^{0} \rightarrow \Xi_{b}^{-}$ & -0.554 & 0.41 & 0.23 & -0.0655 & 0.313 & 0.416 & 0.263 & -0.0857 \\
\hline$\Omega_{b c}^{0} \rightarrow \Omega_{b}^{-}$ & -0.512 & 0.366 & 0.0841 & 0.013 & 2.38 & 0.416 & 0.121 & -0.00663 \\
\hline$\Xi_{b c}^{+} \rightarrow \Sigma_{c}^{++}$ & -0.094 & 0.118 & 0.0027 & $-3.91 \times 10^{-4}$ & 0.124 & 0.232 & -0.0196 & $3.63 \times 10^{-4}$ \\
\hline$\Xi_{b c}^{+\infty} \rightarrow \Xi_{c c}^{++}$ & 0.771 & 0.0531 & 0.00247 & $-1.02 \times 10^{-4}$ & -0.0579 & 0.0459 & $3.56 \times 10^{-4}$ & $1.02 \times 10^{-4}$ \\
\hline$\Xi_{b c}^{0} \rightarrow \Lambda_{c}^{+}$ & -0.104 & 0.194 & -0.0165 & $3.17 \times 10^{-4}$ & -0.0262 & 0.265 & -0.0254 & $5.63 \times 10^{-4}$ \\
\hline$\Xi_{b c}^{0} \rightarrow \Sigma_{c}^{+}$ & -0.0664 & 0.118 & 0.00268 & $-3.9 \times 10^{-4}$ & 0.0875 & 0.232 & -0.0196 & $3.64 \times 10^{-4}$ \\
\hline$\Xi_{b c}^{0} \rightarrow \Xi_{c c}^{+}$ & 0.771 & 0.0531 & 0.00247 & $-1.02 \times 10^{-4}$ & -0.0579 & 0.0459 & $3.56 \times 10^{-4}$ & $1.02 \times 10^{-4}$ \\
\hline$\Omega_{b c}^{0} \rightarrow \Xi_{c}^{+}$ & -0.0944 & 0.203 & -0.0191 & $4.09 \times 10^{-4}$ & -0.0239 & 0.26 & -0.0269 & $6.38 \times 10^{-4}$ \\
\hline$\Omega_{b c}^{0} \rightarrow \Omega_{c c}^{+}$ & 0.745 & 0.0544 & 0.003 & $-1.52 \times 10^{-4}$ & -0.0669 & 0.0497 & 0.00135 & $3.14 \times 10^{-5}$ \\
\hline
\end{tabular}

TABLE VIII. Parameters of form factors $g_{1}$ and $g_{2}$ for $b c$ baryons.

\begin{tabular}{|c|c|c|c|c|c|c|c|c|}
\hline \multirow[b]{2}{*}{$\mathcal{B}_{1} \rightarrow \mathcal{B}_{2}$} & \multicolumn{4}{|c|}{$g_{1}$} & \multicolumn{4}{|c|}{$g_{2}$} \\
\hline & $F(0)$ & $\alpha\left(\mathrm{GeV}^{-2}\right)$ & $\beta\left(\mathrm{GeV}^{-4}\right)$ & $\gamma\left(\mathrm{GeV}^{-6}\right)$ & $F(0)$ & $\alpha\left(\mathrm{GeV}^{-2}\right)$ & $\beta\left(\mathrm{GeV}^{-4}\right)$ & $\gamma\left(\mathrm{GeV}^{-6}\right)$ \\
\hline$\Xi_{b c}^{+} \rightarrow \Lambda_{b}^{0}$ & 0.147 & 0.295 & 0.0589 & -0.0201 & -0.095 & 1.32 & -0.664 & -0.0546 \\
\hline$\Xi_{b c}^{+} \rightarrow \Sigma_{b}^{0}$ & -0.414 & 0.292 & 0.0462 & -0.0124 & 0.174 & 0.816 & 0.194 & -0.479 \\
\hline$\Xi_{b c}^{+} \rightarrow \Xi_{b}^{0}$ & 0.167 & 0.275 & 0.042 & -0.00782 & -0.11 & 1.11 & -0.251 & -0.0448 \\
\hline$\Xi_{b c}^{0 c} \rightarrow \Sigma_{b}^{-}$ & -0.586 & 0.292 & 0.0461 & -0.0123 & 0.247 & 0.814 & 0.199 & -0.482 \\
\hline$\Xi_{b c}^{0} \rightarrow \Xi_{b}^{-}$ & 0.167 & 0.275 & 0.0419 & -0.00779 & -0.111 & 1.08 & -0.225 & -0.0543 \\
\hline$\Omega_{b c}^{0} \rightarrow \Xi_{b}^{-}$ & -0.146 & 0.296 & 0.0642 & -0.0226 & 0.104 & 1.23 & -0.717 & 0.00824 \\
\hline$\Omega_{b c}^{0} \rightarrow \Omega_{b}^{-}$ & -0.67 & 0.272 & 0.0407 & -0.00635 & 0.32 & 0.181 & 1.08 & -0.654 \\
\hline$\Xi_{b c}^{+} \rightarrow \Sigma_{c}^{++}$ & -0.139 & 0.123 & -0.00335 & $-1.2 \times 10^{-4}$ & -0.00321 & 0.171 & 0.0308 & -0.00178 \\
\hline$\Xi_{b c}^{+} \rightarrow \Xi_{c c}^{++}$ & 0.511 & 0.0474 & 0.00162 & $-2.64 \times 10^{-5}$ & -0.0669 & 0.057 & 0.00324 & $-1.53 \times 10^{-4}$ \\
\hline$\Xi_{b c}^{0} \rightarrow \Lambda_{c}^{+}$ & -0.0428 & 0.166 & -0.00808 & $6.56 \times 10^{-6}$ & 0.0194 & 0.17 & -0.0141 & $2.58 \times 10^{-4}$ \\
\hline$\Xi_{b c}^{0} \rightarrow \Sigma_{c}^{+}$ & -0.098 & 0.123 & -0.00336 & $-1.2 \times 10^{-4}$ & -0.00226 & 0.172 & 0.0307 & -0.00178 \\
\hline$\Xi_{b c}^{0} \rightarrow \Xi_{c c}^{+}$ & 0.511 & 0.0474 & 0.00162 & $-2.64 \times 10^{-5}$ & -0.0669 & 0.057 & 0.00324 & $-1.53 \times 10^{-4}$ \\
\hline$\Omega_{b c}^{0} \rightarrow \Xi_{c}^{+}$ & -0.0378 & 0.205 & -0.0143 & $1.97 \times 10^{-4}$ & 0.0186 & 0.181 & -0.017 & $3.59 \times 10^{-4}$ \\
\hline$\Omega_{b c}^{0} \rightarrow \Omega_{c c}^{+}$ & 0.493 & 0.0491 & 0.00209 & $-6.17 \times 10^{-5}$ & -0.0713 & 0.0578 & 0.00424 & $-2.55 \times 10^{-4}$ \\
\hline
\end{tabular}


TABLE IX. Parameters of form factors $f_{1}$ and $f_{2}$ for $b b$ baryons.

\begin{tabular}{|c|c|c|c|c|c|c|c|c|}
\hline \multirow[b]{2}{*}{$\mathcal{B}_{1} \rightarrow \mathcal{B}_{2}$} & \multicolumn{4}{|c|}{$f_{1}$} & \multicolumn{4}{|c|}{$f_{2}$} \\
\hline & $F(0)$ & $\alpha\left(\mathrm{GeV}^{-2}\right)$ & $\beta\left(\mathrm{GeV}^{-4}\right)$ & $\gamma\left(\mathrm{GeV}^{-6}\right)$ & $F(0)$ & $\alpha\left(\mathrm{GeV}^{-2}\right)$ & $\beta\left(\mathrm{GeV}^{-4}\right)$ & $\gamma\left(\mathrm{GeV}^{-6}\right)$ \\
\hline$\overline{\Xi_{b b}^{0} \rightarrow \Sigma_{b}^{+}}$ & -0.0751 & 0.254 & -0.0267 & $6.45 \times 10^{-4}$ & 0.207 & 0.194 & -0.0253 & $7.04 \times 10^{-4}$ \\
\hline$\Xi_{b b}^{0 b} \rightarrow \Xi_{b c}^{+}$ & 0.592 & 0.0674 & 0.00469 & $-4.29 \times 10^{-4}$ & -0.266 & 0.0701 & 0.00503 & $-5.03 \times 10^{-4}$ \\
\hline$\Xi_{b b}^{0 b} \rightarrow \Xi_{b c}^{\prime+}$ & -0.00381 & -0.858 & 0.0343 & 0.0124 & 0.527 & 0.0711 & 0.00515 & $-5.22 \times 10^{-4}$ \\
\hline$\Xi_{b b}^{b D} \rightarrow \Lambda_{b}^{0}$ & -0.101 & 0.168 & -0.0213 & $5.62 \times 10^{-4}$ & $6.04 \times 10^{-4}$ & 0.169 & -0.0218 & $5.83 \times 10^{-4}$ \\
\hline$\Xi_{b b}^{-} \rightarrow \Sigma_{b}^{0}$ & -0.0531 & 0.254 & -0.0267 & $6.45 \times 10^{-4}$ & 0.146 & 0.194 & -0.0253 & $7.03 \times 10^{-4}$ \\
\hline$\Xi_{b b}^{b D} \rightarrow \Xi_{b c}^{0}$ & 0.592 & 0.0674 & 0.00469 & $-4.29 \times 10^{-4}$ & -0.266 & 0.0701 & 0.00503 & $-5.03 \times 10^{-4}$ \\
\hline$\Xi_{b b}^{-} \rightarrow \Xi_{b c}^{\prime 0}$ & -0.00384 & -0.861 & 0.0361 & 0.0122 & 0.527 & 0.0711 & 0.00514 & $-5.22 \times 10^{-4}$ \\
\hline$\Omega_{b h}^{-} \rightarrow \Xi_{b}^{0 c}$ & -0.098 & 0.165 & -0.0216 & $5.84 \times 10^{-4}$ & 0.00121 & 0.167 & -0.0221 & $6.03 \times 10^{-4}$ \\
\hline$\Omega_{b b}^{-} \rightarrow \Xi_{b}^{\prime 0}$ & -0.0527 & 0.248 & -0.0271 & $6.75 \times 10^{-4}$ & 0.146 & 0.186 & -0.0248 & $6.96 \times 10^{-4}$ \\
\hline$\Omega_{b b}^{-b D} \rightarrow \Omega_{b c}^{0}$ & 0.586 & 0.0689 & 0.00465 & $-4.5 \times 10^{-4}$ & -0.27 & 0.0713 & 0.00496 & $-5.23 \times 10^{-4}$ \\
\hline$\Omega_{b b}^{-} \rightarrow \Omega_{b c}^{\prime 0}$ & -0.00302 & -1.0 & 0.032 & 0.0167 & 0.527 & 0.0723 & 0.00508 & $-5.43 \times 10^{-4}$ \\
\hline
\end{tabular}

TABLE X. Parameters of form factors $g_{1}$ and $g_{2}$ for $b b$ baryons.

\begin{tabular}{|c|c|c|c|c|c|c|c|c|}
\hline \multirow[b]{2}{*}{$\mathcal{B}_{1} \rightarrow \mathcal{B}_{2}$} & \multicolumn{4}{|c|}{$g_{1}$} & \multicolumn{4}{|c|}{$g_{2}$} \\
\hline & $F(0)$ & $\alpha\left(\mathrm{GeV}^{-2}\right)$ & $\beta\left(\mathrm{GeV}^{-4}\right)$ & $\gamma\left(\mathrm{GeV}^{-6}\right)$ & $F(0)$ & $\alpha\left(\mathrm{GeV}^{-2}\right)$ & $\beta\left(\mathrm{GeV}^{-4}\right)$ & $\gamma\left(\mathrm{GeV}^{-6}\right)$ \\
\hline$\Xi_{b b}^{0} \rightarrow \Sigma_{b}^{+}$ & -0.116 & 0.208 & -0.022 & $5.26 \times 10^{-4}$ & -0.0231 & 0.122 & -0.015 & $3.78 \times 10^{-4}$ \\
\hline$\Xi_{b b}^{0} \rightarrow \Xi_{b c}^{+}$ & 0.376 & 0.0606 & 0.00387 & $-3.02 \times 10^{-4}$ & -0.0131 & 0.11 & 0.00769 & $-5.88 \times 10^{-4}$ \\
\hline$\Xi_{b b}^{0} \rightarrow \Xi_{b c}^{\prime+}$ & -0.312 & 0.0595 & 0.00368 & $-2.75 \times 10^{-4}$ & -0.0448 & 0.0527 & 0.00293 & $-2.69 \times 10^{-4}$ \\
\hline$\Xi_{b b}^{-} \rightarrow \Lambda_{b}^{0}$ & -0.0336 & 0.204 & -0.0225 & $5.51 \times 10^{-4}$ & 0.0112 & 0.243 & -0.026 & $6.28 \times 10^{-4}$ \\
\hline$\Xi_{b b}^{-} \rightarrow \Sigma_{b}^{0}$ & -0.0822 & 0.208 & -0.022 & $5.26 \times 10^{-4}$ & -0.0163 & 0.122 & -0.015 & $3.77 \times 10^{-4}$ \\
\hline$\Xi_{b b}^{-} \rightarrow \Xi_{b c}^{0}$ & 0.376 & 0.0606 & 0.00388 & $-3.03 \times 10^{-4}$ & -0.0135 & 0.109 & 0.00759 & $-5.81 \times 10^{-4}$ \\
\hline$\Xi_{b b}^{-} \rightarrow \Xi_{b c}^{\prime 0}$ & -0.312 & 0.0594 & 0.00368 & $-2.76 \times 10^{-4}$ & -0.0454 & 0.0528 & 0.00294 & $-2.7 \times 10^{-4}$ \\
\hline$\Omega_{b b}^{-} \rightarrow \Xi_{b}^{0}$ & -0.0331 & 0.204 & -0.0231 & $5.75 \times 10^{-4}$ & 0.0107 & 0.245 & -0.0269 & $6.67 \times 10^{-4}$ \\
\hline$\Omega_{b b}^{-} \rightarrow \Xi_{b}^{\prime 0}$ & -0.0801 & 0.208 & -0.0226 & $5.5 \times 10^{-4}$ & -0.0161 & 0.117 & -0.0149 & $3.84 \times 10^{-4}$ \\
\hline$\Omega_{b b}^{-} \rightarrow \Omega_{b c}^{0}$ & 0.371 & 0.062 & 0.00389 & $-3.09 \times 10^{-4}$ & -0.0152 & 0.111 & 0.00592 & $-5.31 \times 10^{-4}$ \\
\hline$\Omega_{b b}^{-} \rightarrow \Omega_{b c}^{\prime 0}$ & -0.308 & 0.0608 & 0.00368 & $-2.8 \times 10^{-4}$ & -0.0436 & 0.0523 & 0.00304 & $-2.82 \times 10^{-4}$ \\
\hline
\end{tabular}

[1] A. Ocherashvili et al. (SELEX Collaboration), Phys. Lett. B 628, 18 (2005).

[2] R. Aaij et al. (LHCb Collaboration), Phys. Rev. Lett. 119, 112001 (2017).

[3] G. Buchalla, A. J. Buras, and M. E. Lautenbacher, Rev. Mod. Phys. 68, 1125 (1996).

[4] W. Wang, F.-S. Yu, and Z.-X. Zhao, Eur. Phys. J. C 77, 781 (2017).

[5] Y.-J. Shi, W. Wang, and Z.-X. Zhao, arXiv:1902.01092.

[6] M. Tanabashi et al. (Particle Data Group), Phys. Rev. D 98, 030001 (2018).

[7] R. A. Briere et al. (CLEO Collaboration), Phys. Rev. Lett. 90, 181802 (2003).
[8] A. K. Likhoded and A. V. Luchinsky, Yad. Fiz. 76, 838 (2013) [Phys. At. Nucl. 76, 787 (2013)].

[9] D. J. Lange, Nucl. Instrum. Methods Phys. Res., Sect. A 462, 152 (2001).

[10] A. V. Luchinsky, Phys. Rev. D 86, 074024 (2012).

[11] A. V. Berezhnoy, A. K. Likhoded, and A. V. Luchinsky, arXiv:1104.0808.

[12] A. K. Likhoded and A. V. Luchinsky, Phys. Rev. D 82, 014012 (2010).

[13] A. K. Likhoded and A. V. Luchinsky, Phys. Rev. D 81, 014015 (2010).

[14] R. Aaij et al. (LHCb Collaboration), Eur. Phys. J. C 77, 72 (2017). 
[15] V. Khachatryan et al. (CMS Collaboration), J. High Energy Phys. 01 (2015) 063.

[16] R. Aaij et al. (LHCb Collaboration), J. High Energy Phys. 05 (2014) 148.

[17] R. Aaij et al. (LHCb Collaboration), Phys. Rev. Lett. 108, 251802 (2012).
[18] R. Perez-Marcial, R. Huerta, A. Garcia, and M. Avila-Aoki, Phys. Rev. D 40, 2955 (1989); 44, 2203(E) (1991).

[19] C. Albertus, E. Hernandez, and J. Nieves, Proc. Sci., QNP2012 (2012) 073 [arXiv:1206.5612]. 